\title{
THE FREE METABELIAN GROUP OF EXPONENT $p^{2}$
}

\author{
N. D. GUPTA
}

Introduction. For $n \geqq 2$ and $p$ a prime, let $M_{n, p^{2}}$ denote the free metabelian group of exponent $p^{2}$ on $n$ generators. The precise nilpotency class of $M_{n, 4}(n \geqq 2)$ was established in Gupta and Tobin [2], where it was also shown that $\left|M_{2,4}\right|=2^{10}$ and $\left|M_{3,4}\right|=2^{34}$. In a recent paper [1], Bachmuth and Mochizuki have shown that the class of $M_{2, p^{2}}$ ( $p$ odd) is precisely $2\left(p^{2}-p\right.$ ). In another paper (to appear) Bachmuth, Heilbronn and Mochizuki have shown that for $3 \leqq k \leqq p+1$, the class of $M_{k, p^{2}}$ is $2\left(p^{2}-p\right)$ or $2\left(p^{2}-p\right)+1$. In this paper we complete the discussion for the case $k \geqq p+2$.

THEOREM. If $n \geqq p+2$, the class of $M_{n, p^{2}}$ is precisely $n(p-1)+(p-1)^{2}$.

Proof of the theorem. For $n \geqq p+2$, Theorem 3 of Gupta, Newman and Tobin [3] gives $n(p-1)+(p-1)^{2}$ as an upper bound for the nilpotency class of $M_{n, p^{2}}$. In this note we obtain $n(p-1)+(p-1)^{2}$ as a lower bound by exhibiting a metabelian group $G$ on $n$ generators which is of exponent $p^{2}$ and the $\left(n(p-1)+(p-1)^{2}\right)$ th term of whose lower central series is nontrivial.

Let $A=g p\left\{a_{1}, \cdots, a_{n}(n \geqq 2) ; a_{1}^{p^{2}}, a_{i}^{p}(i=2, \cdots, n), a_{i} a_{j}=a_{j} a_{i}\right.$ $(i, j=1, \cdots, n)\}$, and let $Z_{p} A$ be the group ring of $A$ over integers $\bmod p$. Let $J$ denote the ideal of $Z_{p} A$ generated by all polynomials $1+a+\cdots+a^{p^{2}-1}$ for $a \in A$ and let $\hat{J}$ denote the ideal of $Z_{p} A$ generated by $\left(1-a_{1}\right)^{p^{2}-p}$. If $a \in g p\left\{a_{2}, \cdots, a_{n}\right\}$, then

$$
1+a+\cdots+a^{p^{2}-1}=p\left(1+a+\cdots+a^{p-1}\right)=0 .
$$

Thus each generating element of $J$ is of the form $1+a+\cdots+a^{p^{2}-1}$, where $a=a_{1}^{s} a^{*}$ for some $s \in\left\{1, \cdots, p^{2}-1\right\}$ and $a^{*} \in g p\left\{a_{2}, \cdots, a_{n}\right\}$. On the other hand

$$
\begin{aligned}
1+a+\cdots+a^{p^{2}-1} & =\left(1+a+\cdots+a^{p-1}\right)\left(1+a^{p}+\cdots+a^{p(p-1)}\right) \\
& =\rho \cdot\left(1+a_{1}^{s p}+\cdots+a_{1}^{s p(p-1)}\right) \quad\left(\text { where } \rho \in Z_{p} A\right) \\
& =\rho\left(1-a_{1}^{s}\right)^{p(p-1)} \in \hat{J} ;
\end{aligned}
$$

so that $J \subseteq \hat{J}$, and we conclude that

$$
\left(1-a_{1}\right)^{p^{2}-p-1}\left(1-a_{2}\right)^{p-1} \cdots\left(1-a_{n}\right)^{p-1} \notin J .
$$

Let $\left\{\lambda_{1}, \cdots, \lambda_{n}\right\}$ be a set of independent and commuting indeterminates which also commute with every element of $Z_{p} A / J$ and form

Received by the editors November 4, 1968. 
the polynomial ring $R=Z_{p} A / J\left[\lambda_{1}, \cdots, \lambda_{n}\right]$ with coefficients in $Z_{p} A / J$. For $i=1, \cdots, n$, let $a_{i}$ denote the $2 \times 2$ matrix

$$
\left(\begin{array}{ll}
1 & \lambda_{i} \\
0 & a_{i}
\end{array}\right)
$$

over $R$ and let $G$ be the group generated by $a_{i}$ 's under matrix multiplication. By a well-known result of Magnus [4] $G$ is metabelian, and it is easily seen to be of exponent $p^{2}$.

We note that if

$$
a=\left[a_{1}, a_{2}, \cdots, a_{2}, \cdots, a_{n}, \cdots, a_{n}, a_{1}, \cdots, a_{1}\right],
$$

where $a_{2}, \cdots, a_{n}$ repeat $p-1$ times and $a_{1}$ repeats $p^{2}-p-1$ times, then

$$
a=\left(\begin{array}{cc}
1 & \alpha_{1} \lambda_{1}+\alpha_{2} \lambda_{2} \\
0 & 1
\end{array}\right),
$$

where $\alpha_{1}=-\left(1-a_{2}\right)^{p-1} \cdots\left(1-a_{n}\right)^{p-1}\left(1-a_{1}\right)^{p^{2}-p-1}$ and $\alpha_{2}=\left(1-a_{2}\right)^{p-2}$. $\left(1-a_{3}\right)^{p-1} \cdots\left(1-a_{n}\right)^{p-1}\left(1-a_{1}\right)^{p^{2}-p}$.

Since $\lambda_{1}, \lambda_{2}$ are independent,

$$
a=\left(\begin{array}{ll}
1 & 0 \\
0 & 1
\end{array}\right)
$$

if and only if both $\alpha_{1}$ and $\alpha_{2}$ are in $J$. Since $\alpha_{1} \notin J$ by (1), we conclude that $a$ is not the identity element of $G$. Thus the $(n(p-1)+(p-1))$ th term of the lower central series of $G$ is nontrivial and so $G$ has class precisely $n(p-1)+(p-1)^{2}$.

Added IN PROOF. Dr. M. F. Newman has recently proved (written communication) that the bounds obtained in [3] are best possible. Thus our theorem also follows from his general results.

\section{REFERENCES}

1. S. Bachmuth and H. Y. Mochizuki, The class of the free metabelian group with exponent $p^{2}$, Comm. Pure Appl. Math. 21 (1968), 385-399.

2. N. D. Gupta and S. J. Tobin, On certain groups with exponent four, Math. Z. 102 (1967), 216-226.

3. N. D. Gupta, M. F. Newman and S. J. Tobin, On metabelian groups of prime power exponent, Proc. Roy. Soc. Ser. A. 302 (1968), 237-242. 768.

4. W. Magnus, On a theorem of Marshall Hall, Ann. of Math. (2) 40 (1939), 764-

UNIVERSITY OF MANITOBA 